\title{
Guia colombiana para el manejo integral de la estrechez de uretra
}

\section{Colombian Guideline for the Integral Management of the Urethral Stricture}

\author{
Federico Gaviria Gil ${ }^{1}$ Johana Andrea Benavides ${ }^{2}$ Andrés Delgado Montoya ${ }^{2}$
}

${ }^{1}$ Hospital Pablo Tobón Uribe, Medellín, Colombia

${ }^{2}$ Universidad CES, Medellín, Colombia

Urol Colomb 2019;28:193-195.

\section{Introducción}

La estrechez de uretra es definida como la disminución anormal en el calibre de la luz de la uretra que puede estar o no asociado a la fibrosis de los tejidos circundantes (uretra posterior), o del cuerpo esponjoso (uretra anterior). ${ }^{1}$ Esa patología es causada por diferentes condiciones como la inflamación, la infección, trauma o secundario a iatrogenia por diferentes mecanismos y en algunos casos en los cuales no se determina la etiología, se define como idiopática.

La sintomatología no es específica y se puede sobreponer con otras condiciones que incluyen síntomas del tractor urinario inferior (LUTS) e infecciones del tracto urinario (ITU) que pueden confundir el diagnóstico.

\section{Abordaje Inicial}

Los pacientes con estrechez de uretra usualmente consultan por disminución en el calibre del chorro, vaciamiento vesical incompleto, infecciones urinarias, epididimitis, aumento en el residuo postmiccional, retención urinaria, insuficiencia renal, incontinencia o disminución en la fuerza de la eyaculación. También pueden consultar por disuria y cambios en la forma del chorro urinario., ${ }^{2,3}$ Por eso esa entidad se debe tener en cuenta en los diagnósticos diferenciales.

Dentro de los estudios básicos se debe realizar creatinina, un uroanálisis y un urocultivo. En cuanto a las ayudas diagnósticas el estándar de oro en el diagnóstico de la estrechez de uretra es la cisto-uretrografía asociada a la cistoscopia con equipo flexible idealmente. ${ }^{4}$ Para la evaluación adecuada de la uretra se requiere de un periodo de tiempo sin instrumentación del tracto urinario para poder
Address for correspondence Dr. Federico Gaviria Gil, MD, Hospital Pablo Tobón Uribe, Medellín, Colombia (e-mail: federico@une.net.co).

determinar la verdadera severidad de la estrechez de 4 a 12 semanas. $^{5}$

\section{Recomendaciones}

1. Se debe incluir la estrechez uretral dentro de los diagnósticos diferenciales de un hombre con síntomas obstructivos del tracto urinario inferior. (Grado C)

2. En el abordaje de un paciente con sospecha de estrechez uretral, se debe realizar un examen físico y una historia clínica completa. (Principio clínico)

3. Se debe utilizar la cisto-uretrografía y la cistoscopia flexible para hacer el diagnóstico de la estrechez uretral. Ellas se deben realizar de forma combinada e idealmente por el urólogo que va a proceder a la reconstrucción. (Grado C)

4. Se debe planear la intervención de forma electiva para determinar adecuadamente la localización y la longitud de la estrechez uretral. (Opinión de experto)

5. Se recomienda en aquellos pacientes que han tenido manipulación reciente de la vía urinaria; esperar al menos 4 semanas antes de realizar la cistoscopia y cisto-uretrografía. (Opinión de experto)

6. En caso de que un paciente con estrechez de uretra presente retención urinaria se debe realizar una cistostomía para derivar la vía urinaria y posteriormente realizar los estudios. (Opinión de experto)

\section{Uretrotomía Interna Óptica (UIO)}

Es un procedimiento endoscópico, mínimamente invasivo que consiste en el corte a las 12 del reloj en el sitio de la estenosis. Puede realizarse con corte frío o con láser con
DOI https://doi.org/ 10.1055/s-0039-1695018. ISSN 0120-789X. eISSN 2027-0119.
Copyright ( 2019, Sociedad Colombiana License terms de Urología. Publicado por Thieme Revinter Publicações Ltda., Rio de Janeiro, Brazil. Todos los derechos reservados. 
iguales resultados. ${ }^{6-8}$ En pacientes que no son candidatos a uretroplastia se recomienda realizar la UIO con un esquema de dilatación que puede ir desde diario hasta semanal para disminuir las tasas de recurrencia. ${ }^{9}$ No hay un consenso establecido sobre eso.

\section{Recomendaciones}

1. Se debe ofrecer dilatación uretral, UIO o uretroplastia en el tratamiento de la estrechez bulbar menor a $1 \mathrm{~cm}$, sin evidencia de espongiofibrosis importante, de etiología no traumática. (Grado $\mathbf{C}$ )

2. El urólogo debe realizar dilatación o UIO cuando se realice tratamiento endoscópico de la estrechez uretral. (Grado C)

3. Después de una UIO se puede retirar de forma segura la sonda uretral en las primeras 72 horas después del procedimiento si no ha habido complicaciones. (Grado C)

4. En pacientes que no son candidatos a uretroplastia, los urólogos pueden recomendar un plan de dilatación después de una UIO para mantener temporalmente la permeabilidad de la uretra. (Grado $\mathbf{C}$ )

5. Se debe ofrecer uretroplastia, en vez de repetir el manejo endoscópico en paciente con estrechez de uretra anterior recurrente que ha fallado a una dilatación uretral o a la UIO. (Grado C)

6. Si el urólogo no hace uretroplastias debe remitir al paciente a un cirujano con experiencia. (Opinión de experto)

\section{Reconstrucción de Uretra Anterior}

La uretra anterior está formada por el meato uretral, la fosa navicular, la uretra peneana y la bulbar. La estrechez de la uretra bulbar representa el sitio más frecuente seguido de la estrechez en el meato uretral, la uretra peneana y panuretral. ${ }^{3,10}$ Cada uno de los segmentos tiene unas características específicas que hacen que el abordaje quirúrgico sea único en cada uno. ${ }^{11,12}$

\section{Recomendaciones}

1. Se sebe tratar de forma inicial la estrechez del meato uretral o de la fosa navicular con dilatación o con meatotomía. (Principio clínico)

2. Se debe ofrecer uretroplastia a los pacientes con recurrencia de la estrechez del meato uretral o de la fosa navicular. (Grado $\mathbf{C}$ )

3. Se debe ofrecer uretroplastia con interposición de tejidos a los pacientes con estrechez de uretra peneana porque con el tratamiento endoscópico la tasa de recurrencia es muy alta. (Grado C)

4. Se debe ofrecer uretroplastia como tratamiento inicial en pacientes con estrechez de uretra bulbar mayor o igual a $2 \mathrm{~cm}$ debido a la baja tasa de éxito de la UIO y a la dilatación uretral. (Grado $\mathbf{C}$ )

5. Se debe realizar reconstrucción de la uretra cuando es larga o son múltiples segmentos en una etapa o múltiples etapas usando como técnica el injerto de mucosa oral el cual es el tejido de elección, colgajos fasciocutáneos o una combinación de esas técnicas. (Grado C)
6. Se debe ofrecer uretrostomía perineal como opción de tratamiento a largo plazo en pacientes no candidatos a reconstrucción compleja que no deseen uretroplastia. (Grado C)

7. Se debe utilizar como primera opción en la uretroplastia el injerto de mucosa oral. (Opinión de expertos)

8. No se debe realizar uretroplastia de sustitución con materiales sintéticos, aloinjerto o xenoinjerto excepto en protocolos de investigación. (Opinión de experto)

9. No se debe realizar uretroplastia en una etapa con injerto tubularizado. (Opinión de experto)

10. No utilizar piel con vello en la uretroplastia con injerto o colgajo. (Principio clínico)

11. Se podría tomar una biopsia cutánea si se sospecha liquen escleroso y se debe tomar si se sospecha carcinoma primario de uretra. (Principio clínico)

12. En estrechez uretral por liquen escleroso se debe evitar el uso de piel genital para la reconstrucción. (Grado B).

\section{Lesión Uretral Asociada a Trauma}

La lesión de uretra se presenta en el 1,6-25\% de los traumas pélvicos. Se debe sospechar en casos de uretrorragia o estigmas de sangrado reciente en el meato uretral, incapacidad para la micción, globo vesical y posiciones anómalas de la próstata al examen físico.

Otro mecanismo de lesión de la uretra es por trauma contuso (a horcajadas) por compresión de la uretra bulbar contra el pubis con lesión parcial o completa. ${ }^{13}$ En esos casos, al ser diferente el mecanismo del trauma, no es aconsejable el realineamiento primario y se podría realizar un primer intento de paso de sonda vesical que no sea traumático ${ }^{14}$.

\section{Recomendaciones}

1. En pacientes con sospecha de trauma uretral se puede realizar un único intento de paso de sonda y si no es posible se debe derivar la vía urinaria con cistostomía. (Grado C)

2. El estudio inicial debe ser una cisto-uretrografía más cistoscopia para lo cual se debe esperar al menos 4 semanas desde el trauma. (Grado $\mathbf{C}$ )

3. Se recomienda la uretroplastia tardía sobre las técnicas endoscópicas en un período de mínimo de 3-6 semanas. (Recomendación de expertos)

4. La reconstrucción definitiva se debe llevar a cabo una vez el paciente se encuentre estable y tolere la posición de litotomía. (Recomendación de expertos)

\section{Contractura de Cuello Vesical/ Estenosis Uretrovesical}

La contractura del cuello vesical es una complicación bien establecida posterior a intervenciones prostáticas ya sea por patología benigna o maligna. Para el tratamiento existen diferentes regímenes; que van desde el tratamiento conservador a la cirugía endoscópica y reconstrucciones abiertas según el grado de severidad y respuesta a los tratamientos previos. 


\section{Recomendaciones}

1. El cirujano puede manejar la contractura del cuello vesical posterior a un procedimiento prostático endoscópico con dilataciones o uretrotomía interna endoscópica. (Opinión de expertos)

2. El cirujano puede tratar la estenosis de la anastomosis uretrovesical con dilataciones o uretrotomía interna endoscópica. (Grado C)

3. Se recomienda la reconstrucción abierta en contracturas o estenosis uretrovesicales recidivantes. (Grado C)

Conflictos de intereses

Los autores declaran no tener conflictos de interés en relación con este estudio.

\section{Bibliografía}

1 Latini JM, McAninch JW, Brandes SB, Chung JY, Rosenstein D. SIU/ICUD Consultation On Urethral Strictures: Epidemiology, etiology, anatomy, and nomenclature of urethral stenoses, strictures, and pelvic fracture urethral disruption injuries. Urology 2014;83(03, Suppl):S1-S7

2 Bayne DB, Gaither TW, Awad MA, Murphy GP, Osterberg EC, Breyer BN. Guidelines of guidelines: a review of urethral stricture evaluation, management, and follow-up. Transl Androl Urol 2017;6(02):288-294

3 Hong MKH, Murugappan S, Norton SM, Moore EM, Grills R. Male urethral stricture disease in a regional centre: 10 years of experience. ANZ J Surg 2019;89(06):747-751

4 Mahmud SM, El KS, Rana AM, Zaidi Z. Is ascending urethrogram mandatory for all urethral strictures? J Pak Med Assoc 2008;58 (08):429-431

5 Hoy NY, Chapman DW, Rourke KF. Better defining the optimal management of penile urethral strictures: A retrospective comparison of single-stage vs. two-stage urethroplasty. Can Urol Assoc J J Assoc Urol Can 2019 Apr 26

6 Torres Castellanos L, Moreno Bencardino MC, Bravo-Balado A, García Mayorga CA, Vargas Manrique I, Fernández N. Evaluation of the Efficacy and Safety of Laser versus Cold Knife Urethrotomy in the Management of Patients with Urethral Strictures: A Systematic Review and Meta-Analysis of Randomized Clinical Trials. Urol Int 2017;99(04):453-459

7 Yenice MG, Seker KG, Sam E, et al. Comparison of cold-knife optical internal urethrotomy and holmium:YAG laser internal urethrotomy in bulbar urethral strictures. Cent European J Urol 2018;71(01):114-120

8 Zheng X, Han X, Cao D, et al. Comparison between cold knife and laser urethrotomy for urethral stricture: a systematic review and meta-analysis of comparative trials. World J Urol 2019

9 Tammela TL, Permi J, Ruutu M, Talja M. Clean intermittent selfcatheterization after urethrotomy for recurrent urethral strictures. Ann Chir Gynaecol Suppl 1993;206:80-83

10 Astolfi RH, Lebani BR, Krebs RK, et al. Specific characteristics of urethral strictures in a developing country (Brazil). World J Urol 2019;37(04):661-666

11 Horiguchi A. Substitution urethroplasty using oral mucosa graft for male anterior urethral stricture disease: Current topics and reviews. Int J Urol 2017;24(07):493-503

12 Mangera A, Chapple C. Management of anterior urethral stricture: an evidence-based approach. Curr Opin Urol 2010;20(06):453-458

13 Obi AO. Short segment bulbar urethral strictures: Review of 48 cases managed in a resource-poor setting. Niger J Clin Pract 2017; 20(08):1020-1026

14 Jeong SH, Park SJ, Kim YH. Efficacy of urethral catheterisation with a hydrophilic guidewire in patients with urethral trauma for treating acute urinary bladder retention after failed attempt at blind catheterisation. Eur Radiol 2012;22(04):758-764

15 Toro ARL, Gil FG. Usos y abusos de la uretrotomía interna óptica. Rev Urol Colomb. 2014;XXIII(02):140-144 